\title{
Producción más Limpia en el Sector Biotecnológico
}

\section{A Cleaner Production Method for the Biotechnological Field}

\author{
Yanisleidys Hernández Bermúdez
}

\begin{abstract}
In this work we present a cleaner production (CP) method was applied to the production process of Erythropoietin in the biotechnological field. CP production potentials were identified from rapid and complete evaluations made during plant processes. Process areas where improvements in the use of water resources, energy, waste generation and waste disposal could be made were determined. The proposed CP method enabled the increase of environmental innovations of the studied process and in addition it can be applied to other biotechnological institutions with similar characteristics to those of the studied process.
\end{abstract}

Keywords: producción más limpia; innovación medioambiental; biotecnología.

En el presente trabajo se aplicó una metodología de Producción más Limpias (PML) al proceso productivo de la Eritropoyetina del sector Biotecnológico. Identificándose en el mismo, potenciales de PML a partir de una evaluación rápida y otra completa en planta. Se determinaron las áreas del proceso en donde se podían hacer mejoras en el uso de los recursos agua, energía, generación y disposición de residuos. Esta metodología de PML propuesta, posibilitó incrementar la innovación medioambiental del proceso estudiado y a su vez esta puede ser utilizada en otros centros de la biotecnología, con características similares al objeto de estudio.

Keywords: cleaner production; environmental innovation; biotechnology.

Departamento: Salud Organizacional y Gestión Medioambiental. Centro de Inmunología Molecular. Calle 216, esquina I5 Atabey. Playa, La Habana, Cuba. C.P.: I I600. Phone:+537 2717933 extensión 3465. Móvil: +535 3106869. E-mail:yanisleydis@cim.sld.cu

ISSN: 07 I8-2724. (http://www.jotmi.org)

Journal of Technology Management \& Innovation (c) Universidad Alberto Hurtado, Facultad de Economía y Negocios. 


\section{Introducción}

La actividad productiva es uno de los pilares fundamentales del desarrollo económico de un país. Sin embargo, los residuos generados y el excesivo consumo de recursos naturales, pueden constituirse en agentes de deterioro del medio ambiente, restando sustentabilidad al crecimiento económico. Como consecuencia de ello, la dimensión ambiental ha adquirido mayor importancia en el diseño de procesos, en la toma de decisiones de inversión y en la gestión productiva. (Zaror, 2000)

En el sector biotecnológico del Ministerio de Ciencia Tecnología y Medio Ambiente (CITMA) en especial el Polo Científico de La Habana, se han empezado a realizar estudios más profundos relacionados con la temática de Producción más Limpia (PML) con el objetivo de lograr que los procesos sean económicamente sostenibles y más competitivos. En el presente trabajo se aplicó una metodología empresarial en el proceso de producción de la Eritropoyetina Humana Recombinante (EPO-hr) a partir de la utilización de herramientas de PML orientada a incrementar la innovación y la eficiencia del proceso, con incidencia positiva en la calidad del producto y la protección al medioambiente.

\section{Metodología}

\section{Ejecución de una evaluación rápida y completa. Caso de estudio planta de producción de la EPO-hr}

Se realizó una evaluación rápida a partir de un recorrido por las instalaciones de la EPO-hr y se hizo un trabajo de expertos donde intervinieron profesionales y técnicos de las diferentes áreas del proceso. Se elaboró y se aplicó un cuestionario a las siguientes áreas: Sistemas Auxiliares, Plantal, Planta 3, Preparación de Medios y Soluciones, así como el Local del Grupo de Apoyo a la Producción para determinar los puntos de emisión de contaminantes. En la confección del cuestionario se emplearon como guía las hojas de trabajo de las evaluaciones rápidas sugeridas en las herramientas de trabajo de PML (Cleaner Production UNIDO Tool Kit, 2004), ajustándolas a las áreas de estudio, sin incluir los aspectos generales de la empresa ni las áreas administrativas por no ser objeto de esta evaluación. Los datos suministrados de la evaluación rápida se procesaron empleando el programa Ecoinspector 2.I, que está basado en el enfoque de proceso según las normas ISO 9000:200I, con el objetivo de determinar las áreas con mayores potenciales para implementar, de forma exitosa, las opciones de PML.A partir de la información recogida durante la evaluación rápida se decidió realizar una evaluación completa para ampliar la información requerida en el proceso. En la evaluación completa se utilizaron varias herramientas de trabajo como:

- El método estándar para la caracterización físico - químicas de los residuos líquidos de cuatro columnas cromatográficas de la etapa de purificación.de la EPO-hr, las cuales son: cromatografía de afinidad (Blue), cromatografía de seudo-afinidad (Quelato), cromatografía de intercambio iónico $(\mathrm{Q})$ y cromatografía de filtración en gel (Superdex). Los ensayos realizados fueron: $\mathrm{pH}$, demanda química de oxígeno (DQO), demanda bioquímica de oxígeno (DBO5), fósforo total $(\mathrm{Nt})$ y nitrógeno total $(\mathrm{Nt})$. (APHA-AWWAWPCS-WEF, 2000)

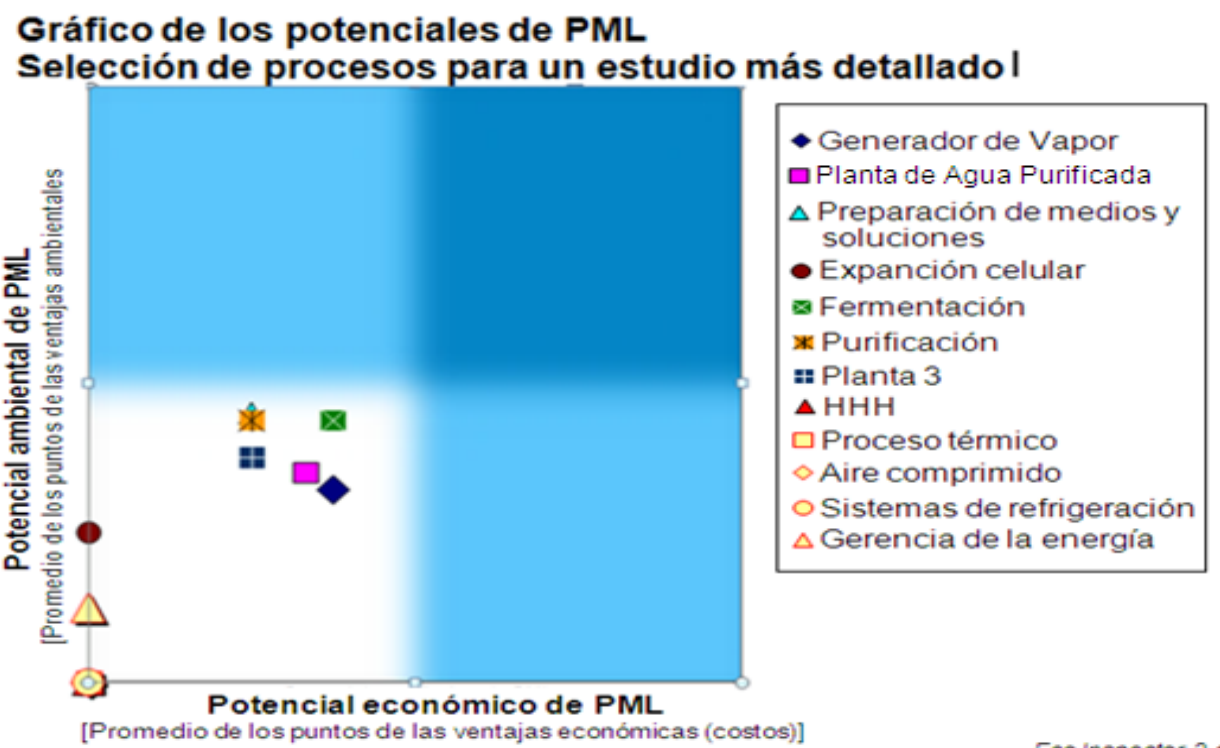

Eco inspector 2.1 - Universidad de Cencias Aplicadas de Basilea (FMBB) 2003

Figura I Potenciales de PML en el proceso de la EPO-hr. (Fuente elaboración propia)

ISSN: 07 I 8-2724. (http://www.jotmi.org)

Journal of Technology Management \& Innovation (c) Universidad Alberto Hurtado, Facultad de Economía y Negocios. 
- La norma IA 6746/I5 para la caracterización de la corriente de rechazo de la osmosis inversa. (Montero, 2002)

- La norma cubana y la brasilera para el diagnóstico de los residuales sólidos. (Ministerio de Ciencia, Tecnología y Medio Ambiente, 2009) (Asociación Brasileña de Normas Técnicas, 1994)

Medidor de gases testo $300 \mathrm{M}-\mathrm{I}$ para determinar el CO contaminante y se estimó las emisiones teóricas de $\mathrm{SO} 2$, a partir del consumo de fuelóleo y la composición de azufre para ello se realizó tres mediciones al generador de vapor pirotubular ENSAC 2,5 t/h y se entraron los datos al programa Contamin. (La O Leyva , 201 I; Reinosa, 2013; Instituto de Investigación de Normalización, 1999)

Una vez identificadas las opciones PML se efectuó un análisis económico, a partir de los costos que pudieran generarse para el estudio y ejecución de las mismas y los beneficios que se pudieran alcanzar.

\section{Resultados}

\section{Resultados de la evaluación rápida en el proceso de la EPO-hr}

A partir de la figura I se observa que con el empleo del software Ecoinspector 2.I la mayor parte de las áreas poseen bajos potenciales de implementación de opciones de PML. Sin embargo, en las áreas de Fermentación, Purificación, Planta 3 (Formulación, Envase y Etiquetado), Generador de Vapor y Planta de Agua Purificada; es posible introducir algunas mejoras, fundamentalmente en los aspectos de aguas de desechos, residuales sólidos y emisiones de los gases de combustión y consumos de energía. En la tabla I se aprecian los problemas y las propuestas de PML.

\section{Resultados de la evaluación completa en el proceso de la EPO-hr}

\section{PML I. Determinación de la composición de los re- siduales líquidos}

En la figura 2 se observa el comportamiento del $\mathrm{pH}$ de los residuales líquidos de las columnas cromatografías estudiadas, predominando valores de $\mathrm{pH}$ de neutro a básicos debido a que la sustancia que se utiliza para la limpieza, higienización y descontaminación de las columnas es el hidróxido de sodio a diferentes concentraciones moleculares. Se destaca que la columna de Superdex está por encima del límite máximo de la planta de lodo activado que trata los residuales del proceso y la columna de Quelato está por debajo del límite mínimo de la misma lo que significa que es necesario homogenizar las corrientes.

En la figura 3 se aprecia que, el punto Blue es el que aporta más DBO5 y DQO de todos los puntos estudiados, ya que recibe el sobrenadante de fermentación, con un alto contenido de carga microbiológica. En general, los parámetros de DBO5 y DQO no cumplen con los límites establecidos por la planta de residuales, lo cual no tendría un gran impacto en la misma ya que el flujo promedio del proceso es de I,45 $\mathrm{m} 3 /$ día y el consumo promedio de la planta es de $700 \mathrm{~m} 3 /$ día. Esto nos indica la necesidad de un tratamiento previo como la inactivación de los residuales y teniendo en cuenta que es un proceso biotecnológico, esto es un requisito necesario.

En la figura 4 se observa que el valor de $\mathrm{Nt}$ de la columna de Blue, están por encima del límite permisible de la planta de tratamiento, esto resultó lógico debido a que en este paso se procesa el sobrenadante de la etapa de fermentación y el punto de $Q$ es el de menor valor de nitrógeno por ser

\begin{tabular}{|l|l|l|}
\hline Áreas & Problemas & Propuesta PML \\
\hline Planta de EPO-hr & & \\
\hline $\begin{array}{l}\text {-Preparación de medios y soluciones. } \\
\text {-Expansión celular } \\
\text {-Fermentación } \\
\text {-Purificación } \\
\text {-Grupo de apoyo a la producción. } \\
\text {-Planta de procesamiento final. }\end{array}$ & $\begin{array}{l}\text {-No se encuentran actualizados los estudios } \\
\text { de caracterización de los residuales líquidos } \\
\text { y sólidos. } \\
\text {-Altos consumos de energía. }\end{array}$ & $\begin{array}{l}\text { • Caracterización de los desechos. } \\
\text { lodo activado puede asimilar los residuales } \\
\text { del proceso de producción de la EPO-hr. }\end{array}$ \\
\hline Sistemas auxiliares de la planta EPO-hr & $\begin{array}{l}\text { No se encuentra actualizado el estudio de } \\
\text { emisión de gases en la chimenea del gener- } \\
\text { ador de vapor. }\end{array}$ & $\begin{array}{l}\text { Realizar el estudio de gases de emisión } \\
\text { contaminantes en el sistema de generador } \\
\text { de vapor. }\end{array}$ \\
\hline -Sistema de generador de vapor. & $\begin{array}{l}\text { Gran volumen de desecho en la unidad de } \\
\text { ósmosis inversa. }\end{array}$ & $\begin{array}{l}\text { Caracterización de la corriente de } \\
\text { desecho. } \\
\text { - Estudiar variantes de reuso interno. }\end{array}$ \\
\hline Sistema de agua purificada. & &
\end{tabular}

Tabla I Propuestas de opciones de PML. Fuente elaboración propia.

ISSN: 07 I8-2724. (http://www.jotmi.org)

Journal of Technology Management \& Innovation (c) Universidad Alberto Hurtado, Facultad de Economía y Negocios. 


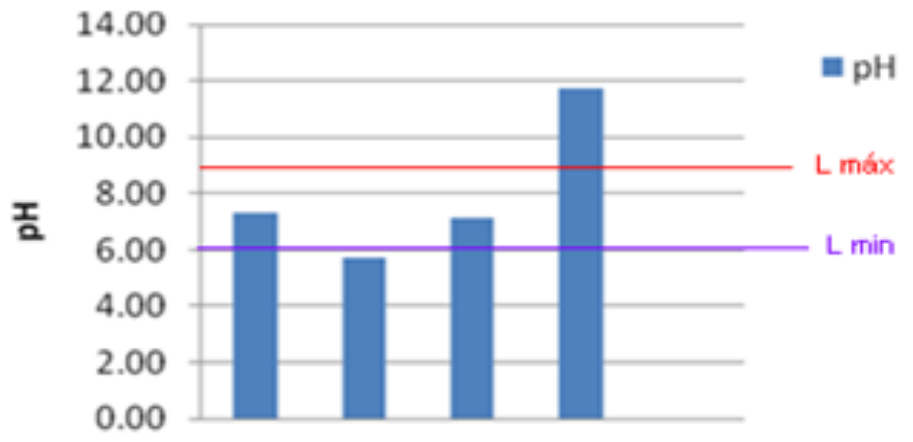

Blue Quelato Q Superdex

Figura 2 Comportamiento del pH en los puntos de muestreo. (Fuente elaboración propia)

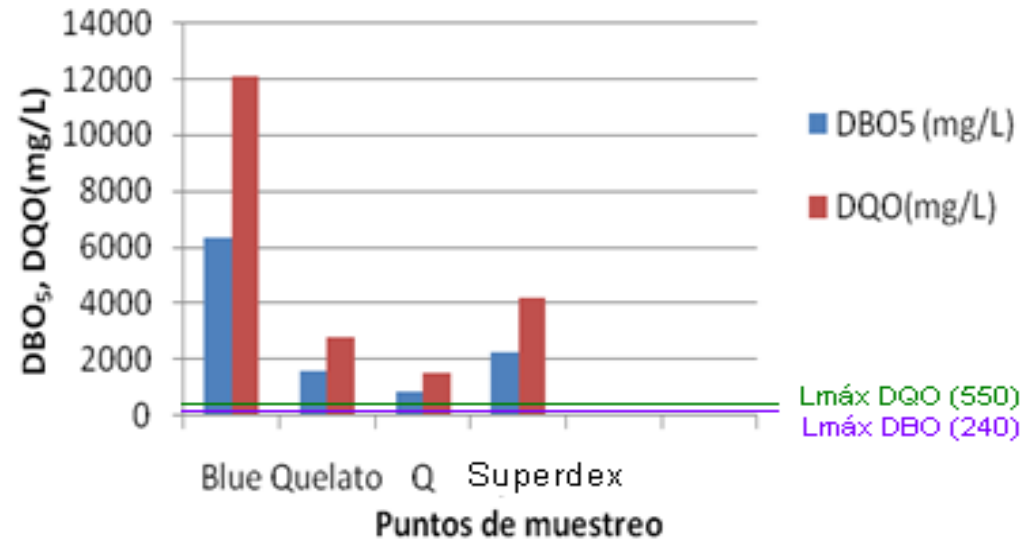

Figura 3 Comportamiento de la DBO5, DQO (Fuente elaboración propia)

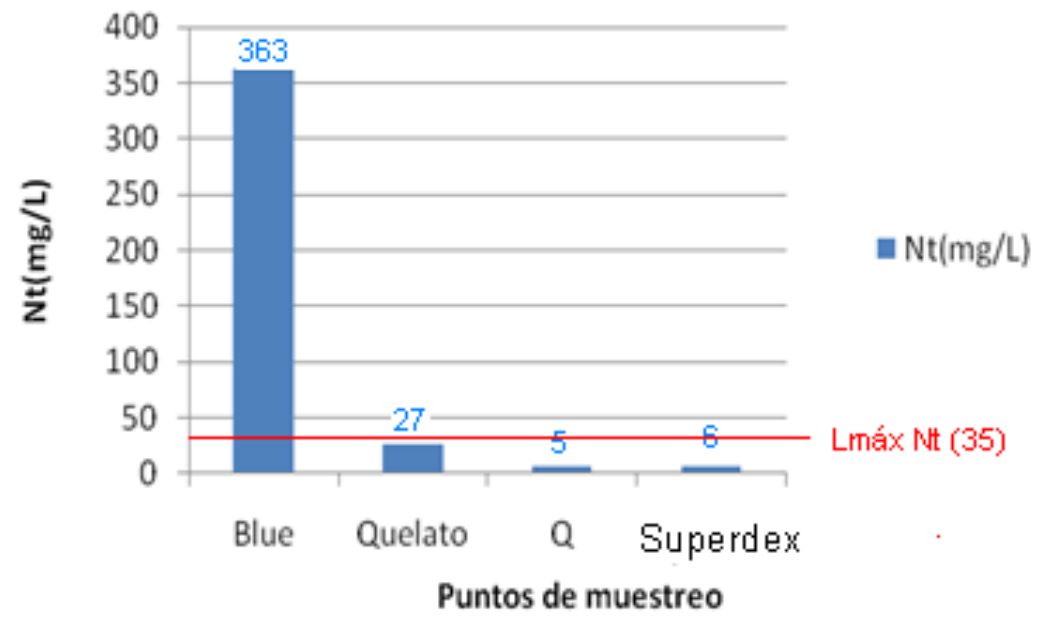

Figura 4 Comportamiento del nitrógeno total. (Fuente elaboración propia) 
el último paso de separación con más de un $98 \%$ de pureza. Este resultado confirma que es necesario compensar el residual.

En la figura 5 se representa el comportamiento del fósforo total en los diferentes puntos de muestreo $y$ en ella se observa que la columna de Quelato es el de mayor valor de Pt. ya que en este paso la elución entra con una solución de hidrógeno fosfato de sodio (Na2HPO4) y la última columna, la Súperdex, es la que menor valor de Pt tiene pues en ella no se emplean soluciones de fosfato en ninguna de sus etapas. Se aprecia que para todas las etapas de separación los valores de $\mathrm{Pt}$ de las columnas, están por debajo del límite permisible de la planta del tratamiento.

En la tabla 2 se reportan las contribuciones de los diferentes puntos de muestro en cuanto a cargas DBO5, DQO totales y la relación entre ellas. Se refleja en todos los casos que la relación $\mathrm{DBO} / \mathrm{DQO}$ se encuentra por encima de 0,5 reafirmándose la biodegradablidad del residual en estos puntos y además indica que el tipo tratamiento a seguir es el biológico ya que la relación de DBO5/DQO es mayor que 0,4 (Pozo, 2009), por lo que se corresponde con el tratamiento que se lleva a cabo en la planta de tratamiento de lo activado. Se evidencia que el paso cromatográfico Quelato es el más biodegradable con un valor de 0,57.

\section{Evaluación técnica medioambiental de la sustitución de columnas de exclusión molecular por filtros tan- genciales}

Se evaluó la posible sustitución de las columnas cromatográficas de Superdex y G-25 por la filtración tangencial (FFT) y se calcularon los consumos de soluciones para cada una de las opciones, demostrándose las ventajas del reemplazo de las columnas cromatográficas de exclusión molecular (G-25 y Superdex) por la FFT. Se observan ventajas operacionales y beneficios medio ambientales como son: la disminución del tiempo de operación, limpieza o higienización (ver tabla 3 ), la reducción de residuos así como un menor empleo de soluciones de limpieza (hidróxido de sodio a diferentes molaridades) y en consecuencia un menor consumo de materias primas. En la figura 6 se muestra el consumo de las soluciones donde se evidencia la reducción de los volúmenes, al utilizar la opción de la filtración tangencial en cada variante. Se puede observar que el mayor consumo de soluciones aparece en el paso de cambio de solución tampón con la G-25, lo cual se debe a que es una de las primeras etapas de purificación del proceso.

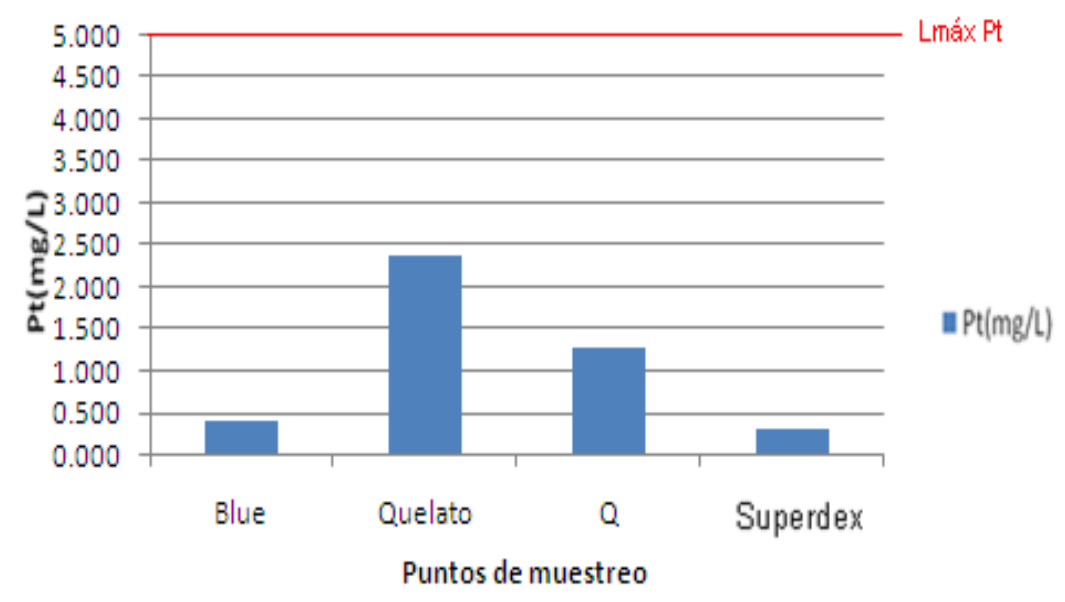

Figura 5 Comportamiento del fósforo total. (Fuente elaboración propia)

\begin{tabular}{|l|l|l|l|}
\hline Puntos & $\mathrm{DQO}(\mathrm{kg} / \mathrm{dia})$ & $\mathrm{DBO}_{5}(\mathrm{~kg} /$ día $)$ & $\mathrm{DBO}_{5} / \mathrm{DQO}$ \\
\hline Blue & 43,64 & 23 & 0,53 \\
\hline Quelato & 3,38 & $\mathrm{I}, 93$ & 0,57 \\
\hline Q & $\mathrm{I}, 18$ & 0,63 & 0,53 \\
\hline Superdex & 0,91 & 0,48 & 0,53 \\
\hline Total & $49,1 \mathrm{I}$ & 26,04 & 0,54 \\
\hline
\end{tabular}

Tabla 2.Valores de las cargas y relación entre la DBO5 /DQO. Fuente elaboración propia.

ISSN: 07 I8-2724. (http://www.jotmi.org) 


\begin{tabular}{|l|l|l|l|l|}
\hline Variables operacionales & $\begin{array}{l}\text { Variante de proceso } \\
\text { G25 }\end{array}$ & $\begin{array}{l}\text { Variante de proceso } \\
\text { FFT }\end{array}$ & $\begin{array}{l}\text { Variante de } \\
\text { Superdex }\end{array}$ & $\begin{array}{l}\text { Variante de proceso } \\
\text { FFT }\end{array}$ \\
\hline Volumen a operar $(\mathrm{L})$ & 60 & 60 & 5 & 5 \\
\hline Tiempo de aplicación $(\mathrm{h})$ & 5,3 & 0,78 & 8,3 & $\mathrm{I}, 05$ \\
\hline $\begin{array}{l}\text { Tiempo de higienización o } \\
\text { limpieza (h) }\end{array}$ & 18,2 & 2 & 5,03 & 2 \\
\hline Tiempo total $(\mathrm{h})$ & 23,5 & 2,78 & 13,33 & 3,05 \\
\hline & & & & \\
\hline
\end{tabular}

Tabla 3 Tabla de tiempos de proceso para cada variante. (Fuente elaboración propia)

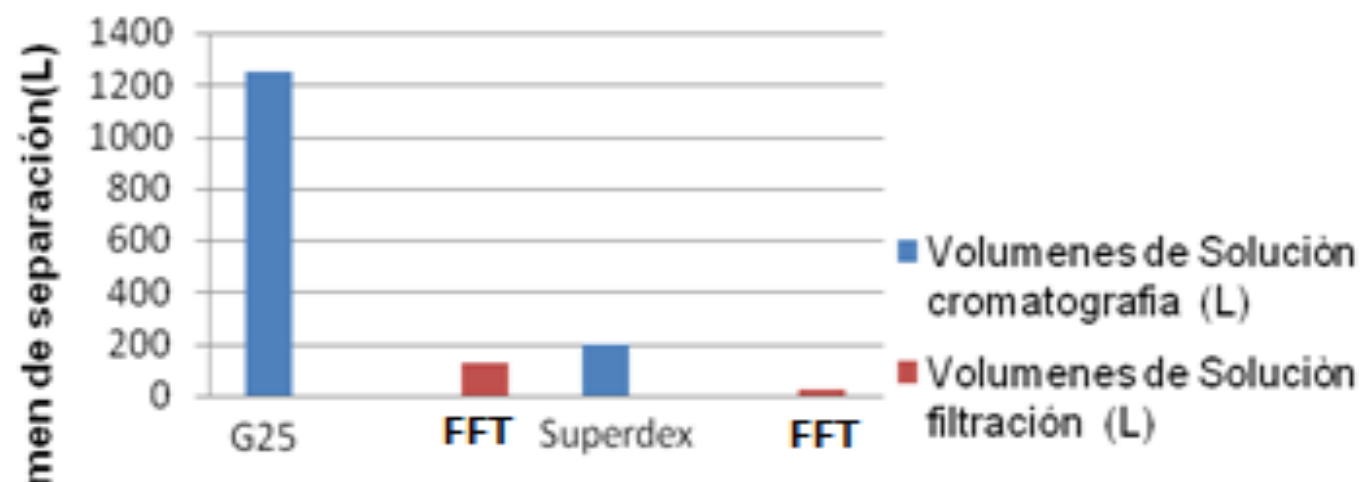

\section{Métodos de separación}

Figura 6 Gráfico de métodos de separación vs volúmenes de solución. (Fuente elaboración propia)

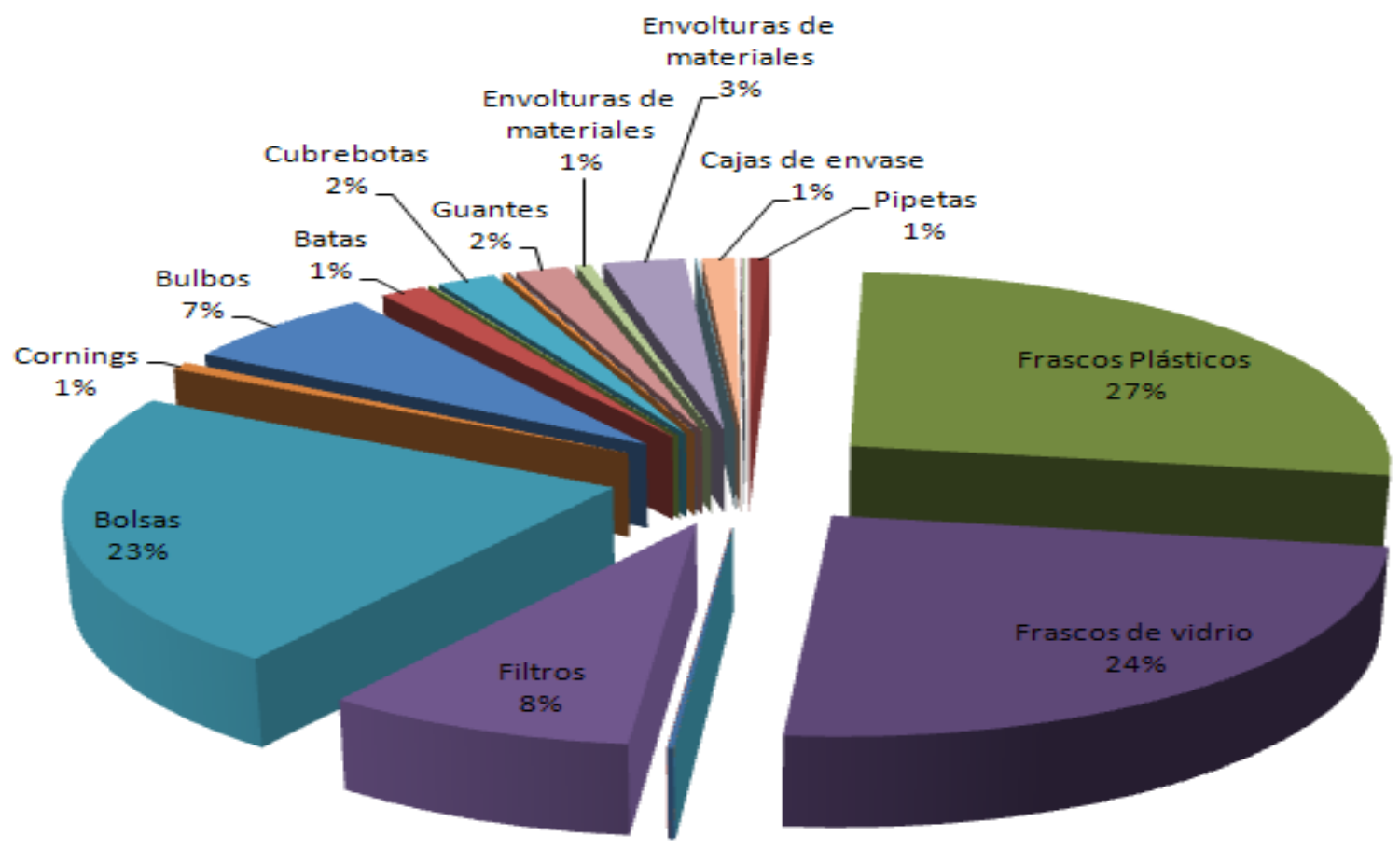

Figura 7 Distribución de residuos totales del proceso de producción de Eritropoyetina. (Fuente elaboración propia) 
PML 2.Clasificación y manejo de los residuales sólidos en el proceso

Se realizó la contabilización de los residuos generados tomando como referencia el punto más crítico de generación de residuales que coincide con el periodo en el que la producción es más intensa. Debido a que el tiempo de generación de cada desecho es diferente, se decidió tomar como base de conteo un año de producción. En la figura 7 se aprecia el \% en peso que representa el peso total de cada residuo en el total de desechos generados, que resultó ser de 30 ton /año. Existen algunos desechos cuyo peso es muy pequeño respecto al total, debido a esto, en el gráfico solo aparecen referenciados aquellos desechos con valor mayor de $1 \%$ en peso. Este gráfico muestra que los residuos que mayor volumen ocupan son las bolsas, los filtros, los frascos plásticos y los frascos y bulbos de vidrio. De aquí se deduce que los principales materiales a tratar son el plástico y el vidrio.

En la tabla 4 se muestran las cantidades de cada material y se evidencia que el residual plástico es el que predomina (20400 $\mathrm{kg} / \mathrm{año}$ ) y el papel y cartón el que menos con $300 \mathrm{~kg} / \mathrm{año}$. En la figura 8 se observa el \% sobre el total que representa cada uno de ellos siendo el plástico y el vidrio la mayor parte de los residuos generados por la EPO-hr, ya que representa el $68 \%$ y un $31 \%$ respectivamente. En el caso del plástico sólo el $9 \%$ es para incinerar y el resto puede ser recuperado.

\section{Propuesta de sistema de gestión de residuos sólidos}

En los locales del proceso como: Expansión Celular (EC), Preparación de Medios y Soluciones (PML), Fermentación (F), Purificación (P), Grupo de Apoyo a la Producción (GAP), Pasillo al Área Limpia (PAL) y Local de Almacenamiento Final (LAF); están habilitados contenedores y bolsas necesarias para el almacenamiento primario, aunque la recolección, el transporte interno y el almacenamiento final, no se efectúan de la manera más adecuada, siendo necesaria la segregación de los desechos en cada una de las áreas. En la tabla 5 se hace una propuesta de tipos de contenedores identificados por colores para establecer el sistema de gestión integral de residuos en el proceso, tomándose como base el trabajo realizado por instalaciones hospitalarias de la Ciudad de Lima en Perú. (Ministerio de Salud, 2004). Se debe evitar el flujo cruzado entre los residuales sólidos generados y los materiales estériles de proceso. En las áreas limpias se debe realizar el saneamiento de los residuos sólidos generados de la jornada anterior, y una vez que se vayan produciendo

\begin{tabular}{|l|l|}
\hline Materiales & Cantidades (Kg/año) \\
\hline Vidrio & 9300 \\
\hline Papel y cartón & 300 \\
\hline Plástico total & 20400 \\
\hline Plástico incinerar & 1836 \\
\hline Plástico recuperar & 18564 \\
\hline
\end{tabular}

Tabla 4 Cantidad de residuos por material generados al año. (Fuente elaboración propia)

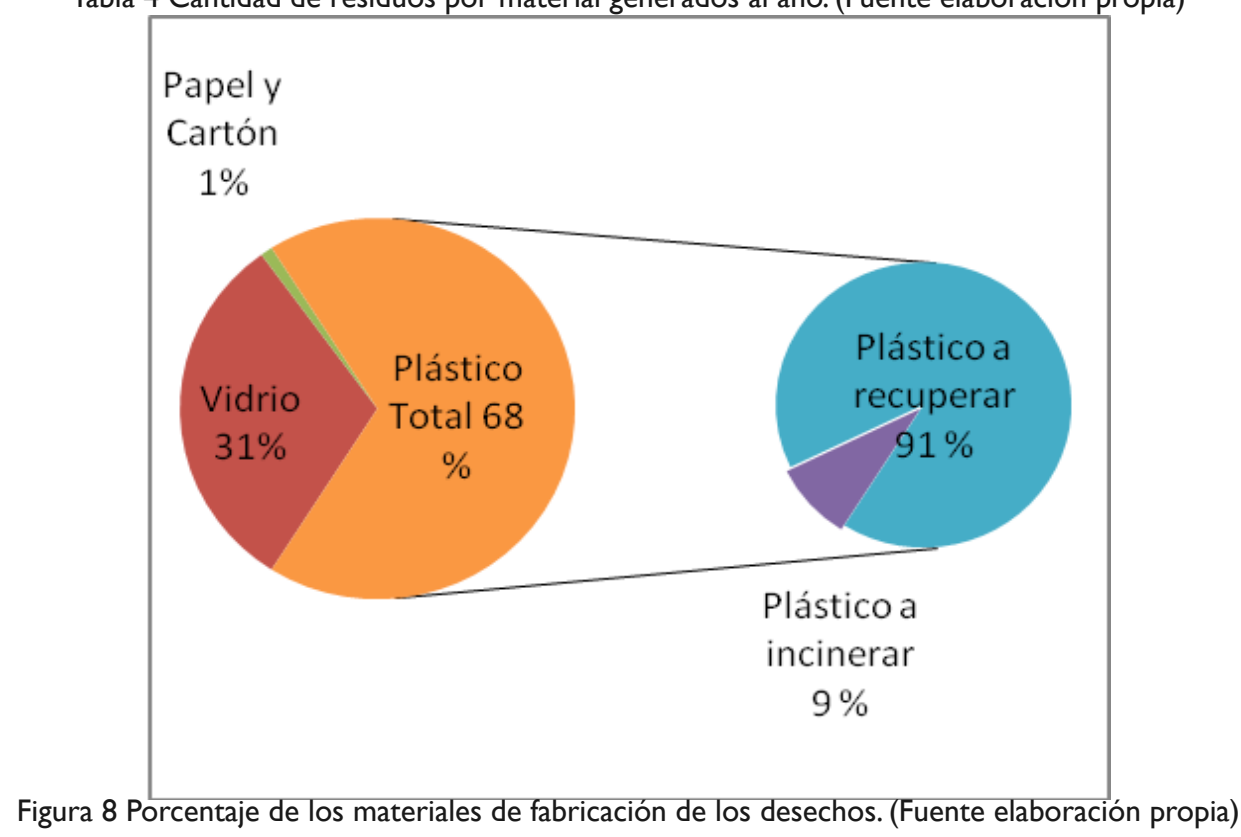

ISSN: 07 I 8-2724. (http://www.jotmi.org)

Journal of Technology Management \& Innovation (c) Universidad Alberto Hurtado, Facultad de Economía y Negocios. 
en el transcurso del día depositarlos en los medios disponibles, logrando que el tiempo de almacenamiento sea el más corto posible para mantener las condiciones ambientales de las áreas. El tamaño de los contenedores en cada local dependerá de la cantidad de desechos generados. En la tabla 6 se puede observar el destino final que se le puede dar a cada material. La mayoría de los residuos son reciclables o reutilizables, siendo una menor porción los residuos que requieren tratamiento.

Se estudió la variante de proponer un ecoincinerador que son equipos quemadores con opciones para hacer menos

\begin{tabular}{|l|l|l|l|l|l|l|l|l|l|}
\hline Tipo de contenedores & EC & PMS & F & P & PPF & GAP & PAL & LAF & Total \\
\hline $\begin{array}{l}\text { Contenedor para plásticos recicla- } \\
\text { bles de color verde. }\end{array}$ & I & I & I & I & I & I & I & I & 8 \\
\hline $\begin{array}{l}\text { Contenedor para plásticos a incin- } \\
\text { erar de color rojo. }\end{array}$ & I & I & I & I & I & I & - & I & 7 \\
\hline $\begin{array}{l}\text { Contenedor para cartón y materi- } \\
\text { ales de oficina de color negro. }\end{array}$ & I & I & I & I & I & I & - & I & 7 \\
\hline $\begin{array}{l}\text { Contenedor para vidrios recicla- } \\
\text { bles de color azúl. }\end{array}$ & - & I & - & - & I & I & - & I & 4 \\
\hline Total por áreas & 3 & 4 & 3 & 3 & 4 & 4 & I & 4 & 26 \\
\hline
\end{tabular}

Tabla 5 Propuesta de cantidades y tipos de contenedores por área. (Fuente elaboración propia)

\begin{tabular}{|c|c|c|}
\hline Materiales & Destino final & Observaciones \\
\hline $\begin{array}{l}\text { I. Envases plásticos contenedores de reactivos } \\
\text { como pueden ser pipetas y ámpulas. }\end{array}$ & $\begin{array}{l}\text {-Realizar un lavado adecuado. } \\
\text {-Entregarlos a la Empresa de Recuperación } \\
\text { de Materia Prima de La Habana. (Cárdenas y } \\
\text { Tamayo, 20I0) }\end{array}$ & $\begin{array}{l}\text { Excepto los envases que con- } \\
\text { tienen cobre que se consideran } \\
\text { residuos peligrosos según el } \\
\text { fabricante. }\end{array}$ \\
\hline $\begin{array}{l}\text { 2. Cartón, papel, guantes de látex, gorros, cu- } \\
\text { brebotas y cubrecalzado y tela desechados. }\end{array}$ & $\begin{array}{l}\text {-Entregarlos a la Empresa de Recuperación } \\
\text { de Materia Prima de La Habana. (Cardenas y } \\
\text { Tamayo 20I0) }\end{array}$ & - \\
\hline $\begin{array}{l}\text { 3. Envases de vidrio contenedores de reacti- } \\
\text { vos. }\end{array}$ & $\begin{array}{l}\text {-Entregarlos a la Empresa de Recuperación } \\
\text { de Materia Prima de La Habana. (Cárdenas y } \\
\text { Tamayo, 20I0) }\end{array}$ & - \\
\hline 4. Bulbos de vidrio & $\begin{array}{l}\text {-Destruirlos a través de un proceso de tritura- } \\
\text { ción. }\end{array}$ & - \\
\hline 5. Agujas usadas en el proceso & $\begin{array}{l}\text {-Depositarlas en una solución de hipoclorito de } \\
\text { sodio al I\%, en recipientes rígidos con un rotu- } \\
\text { lado que indique el tipo de residuo que contiene } \\
\text { y posteriormente llevar a cabo la fundición las } \\
\text { mismas. }\end{array}$ & $\begin{array}{l}\text {-Las agujas utilizadas nunca deben } \\
\text { reciclarse. } \\
\text {-Se debe tapar la aguja empleando } \\
\text { la técnica de una sola mano }\end{array}$ \\
\hline 6. Los filtros y las bolsas provenientes de PMS & $\begin{array}{l}\text {-Realizar un lavado adecuado de las bolsas } \\
\text { y entregarlos a la Empresa de Recuperación } \\
\text { de Materia Prima de La Habana (Cárdenas y } \\
\text { Tamayo, 2010) o donárselas a la Facultad de } \\
\text { Ingeniería Química. }\end{array}$ & $\begin{array}{l}\text { Estas bolsas y filtros contienen } \\
\text { generalmente restos de solu- } \\
\text { ciones de la producción a difer- } \\
\text { entes molaridades, entre otras } \\
\text { sustancias como agua purificada, } \\
\text { alcohol, etc. }\end{array}$ \\
\hline $\begin{array}{l}\text { 7. Los materiales plásticos como: bolsas, filtros, } \\
\text { pipetas y otros que han estado en contacto } \\
\text { con microorganismos genéticamente tratados. }\end{array}$ & Tratamiento térmico e incineración. & $\begin{array}{l}\text { El material de las bolsas es a base } \\
\text { de etileno y propileno, altamente } \\
\text { inflamables y explosivos, pero con } \\
\text { un mínimo impacto ambiental } \\
\text { (Syanez, 2009) }\end{array}$ \\
\hline 8. Contenedores de cobre. & Tratamiento térmico e incineración. & - \\
\hline
\end{tabular}

Tabla 6 Propuesta de destino final de cada material. (Fuente elaboración propia)

ISSN: 07 I8-2724. (http://www.jotmi.org)

Journal of Technology Management \& Innovation (C) Universidad Alberto Hurtado, Facultad de Economía y Negocios. 
contaminantes los gases desprendidos en el proceso de quemado de los residuos. La empresa canadiense ECO WASTE SOLUTIONS (EWS) posee modelos de este tipo de equipo, los cuales son de fácil transporte e instalación, incineran con gases menos contaminantes y tienen bajos costos de operación y mantenimiento. Basándose en esto y en las características de los desechos, se determinó que la opción a escoger para su futura implementación sería la incineración. El modelo CA MODEL CA-50 de la Empresa EWS, está capacitado para incinerar $10,9 \mathrm{~kg} / \mathrm{h}$.A pesar de que es un equipo costoso y que las cantidades que se generan de desechos sólidos para incinerar en una etapa productiva son mínimas de $1836 \mathrm{~kg}$, se considera que se debe comprar el mismo para dar servicios a otras instituciones del Polo Científico de la Habana en el cual existen varios procesos que generan desechos y presentan problemas con la gestión.

\section{PML 3. Recuperación de la corriente de rechazo de la ósmosis inversa}

Se realizaron las mediciones de las características físicoquímicas del agua de rechazo de la ósmosis inversa en un laboratorio especializado. En la tabla 7 se muestran algunos datos de la composición del agua para uso agrícola y los valores de las muestras del agua de rechazo de la ósmosis inversa. Se observa que los valores de los componentes están por debajo de los recomendados para la actividad de uso agrícola, por lo que se puede utilizar este recurso para el riego de las áreas verdes aledaña a la planta de la EPO-hr. El consumo de agua para riego de áreas verdes es de aproximadamente $10 \mathrm{~L} / \mathrm{m} 2$ día (Lobato, 2002), teniendo en cuenta que se dispone de unos $2000 \mathrm{~m} 2$ de jardines, se requeriría de 20000 L /día de agua para estas labores, lo que significa un ahorro considerable si se emplea el agua de rechazo de

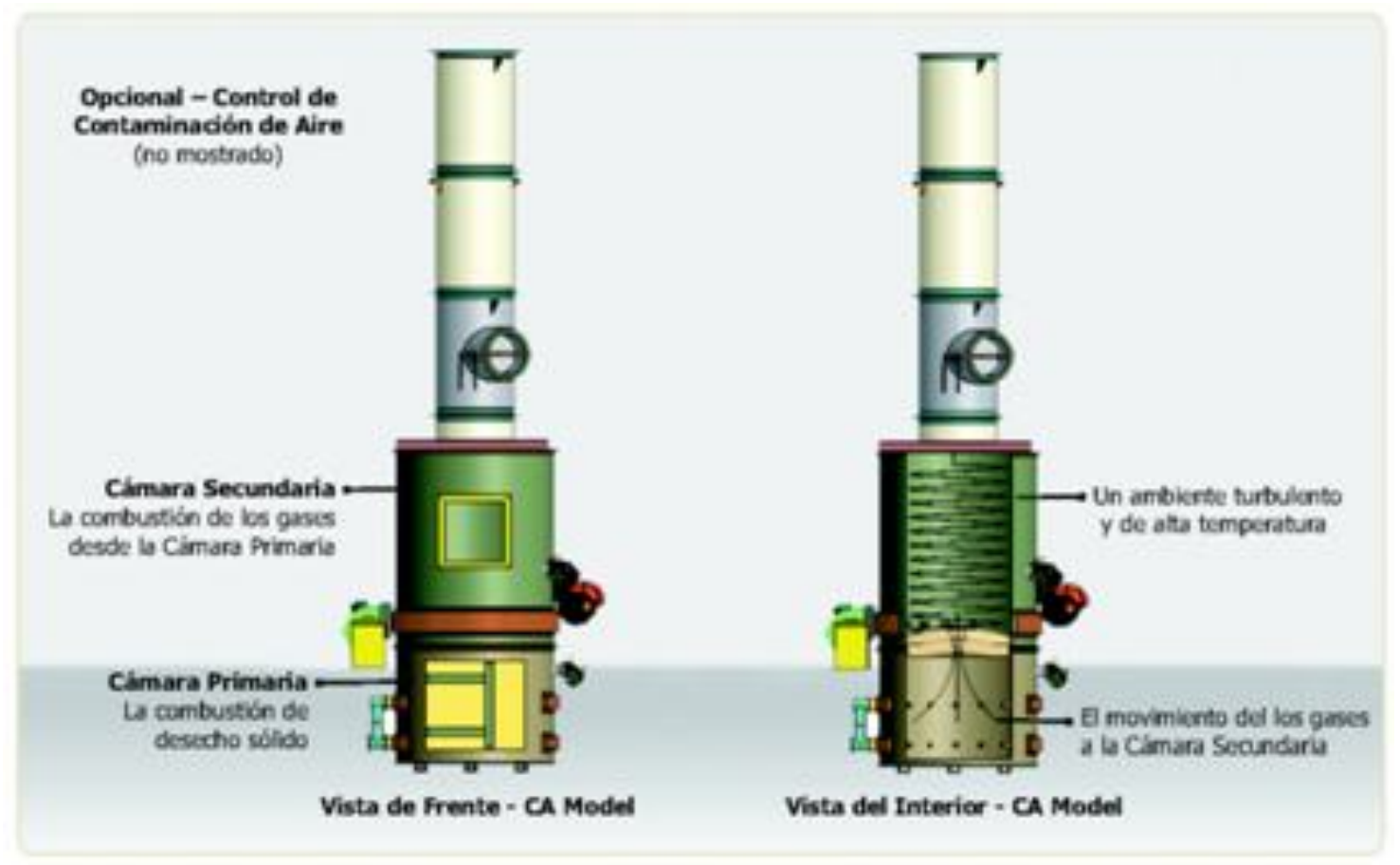

Figura 9 Incinerador ecológico ECO MODEL. (Eco Waste Solutions Process, 2010)

\begin{tabular}{|l|l|l|}
\hline Componentes & Valor medido & Valor máximo recomendado \\
\hline Fe total $(\mathrm{mg} / \mathrm{L})$ & $0.0056 \pm 0.0008$ & 5,0 \\
\hline $\mathrm{Co}(\mathrm{mg} / \mathrm{L})$ & $0.0019 \pm 0.0004$ & 0,05 \\
\hline $\mathrm{Cu}(\mathrm{mg} / \mathrm{L})$ & $0.0104 \pm 0.0010$ & 0,2 \\
\hline $\mathrm{Zn}(\mathrm{mg} / \mathrm{L})$ & $0.0020 \pm 0.0005$ & 2,0 \\
\hline
\end{tabular}

Tabla 7 Composición del agua para uso agrícola y los valores de las muestras del agua de rechazo de la ósmosis inversa. Fuente elaboración propia. (Wikipedia, 2009)

ISSN: 07 I8-2724. (http://www.jotmi.org) 
la ósmosis inversa. Se propone conducir el agua de rechazo de la ósmosis inversa a una pequeña cisterna de $27 \mathrm{~m} 3$ que está en desuso, ver figura 10.

\section{PML 4. Determinación de las emisiones de contami- nantes en el generador de vapor}

Los resultados de la determinación del $\mathrm{CO}$ aparecen reflejados en la tabla 8 y los de la estimación teórica del $\mathrm{SO} 2$ en la tabla 9, en ambos caso se observa que la máxima concentración $(\mathrm{Cma})$ obtenida está muy por debajo del límite permisible que para el caso del $\mathrm{CO}$ es de 5000 y para el $\mathrm{SO} 2$ es de 500 (Norma 39, 1999). Es de destacar que para estos análisis solamente se tuvieron en cuenta las concentraciones de la fuente emisora en estudio, los cuales pudieran variar con la determinación de los niveles de contaminación de fondo de los contaminantes analizados en la zona a la que hacemos referencia.

\section{Costos y ahorros totales}

En la tabla 10 se observa los costos y ahorros en cada una de las alternativas de PML estudiadas. Se observa que la implementación de las opciones de PML identificadas implicaría beneficios ya que se logrará disminuir los costos y los impactos medioambientales negativos en el proceso de la EPO-hr y sus sistemas auxiliares.

\section{Conclusiones}

I. La aplicación del programa Ecoinspector 2.I en la evaluación rápida, identificó en el proceso de la EPO-hr puntos con bajos potenciales de PML, pero en los cuales es posible realizar mejoras.

2. A partir de la realización de la evaluación completa en la planta de la EPO-hr y sus sistemas auxiliares se identificaron 4 potenciales de PML cuyos resultados son los siguientes:

-La relación de DBO5/DQO en todas las columnas cromatografías estudiadas es mayor de 0,5 indicando la biodegradabilidad del residual por lo que el tratamiento final en la planta de lodo activado es adecuado.

-Con la sustitución de las columnas cromatográficas G-25 Blue y Superdex por filtración tangencial se puede ahorrar el $48,4 \%$ de soluciones químicas que representa 700 L/año.

-El 91\% de los residuales plásticos que representa 18564 kg/año del proceso de la EPO-hr se pueden entregar para

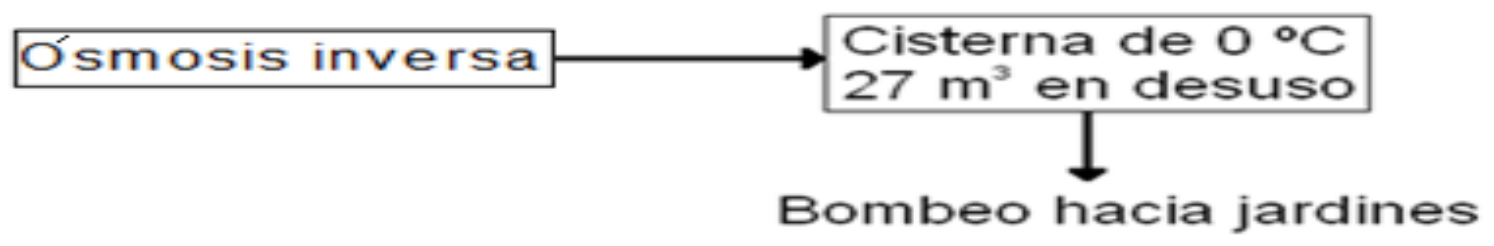

Figura 10 Recuperación de la corriente de rechazo de la ósmosis inversa. Fuente elaboración propia.

\begin{tabular}{|l|l|}
\hline Parámetros & Contamin \\
\hline $\mathrm{I} 2 / 03 / 2010$ & \\
\hline $\mathrm{Cm}\left(\mu \mathrm{g} / \mathrm{m}^{3}\right)$ & $10,0 \mathrm{I}$ \\
\hline Cma $\left(\mu \mathrm{g} / \mathrm{m}^{3}\right)$ & 5000 \\
\hline Dist. Max. Conc. $(\mathrm{m})$ & 156,8 \\
\hline $\mathrm{I} 6 / 04 / 2010$ & \\
\hline $\mathrm{Cm}\left(\mu \mathrm{g} / \mathrm{m}^{3}\right)$ & 2,49 \\
\hline Cma $\left(\mu \mathrm{g} / \mathrm{m}^{3}\right)$ & 5000 \\
\hline Dist. Max. Conc. $(\mathrm{m})$ & 161 \\
\hline $8 / 06 / 2010$ & \\
\hline Cm $\left(\mu \mathrm{g} / \mathrm{m}^{3}\right)$ & 3,08 \\
\hline Cma $\left(\mu \mathrm{g} / \mathrm{m}^{3}\right)$ & 5000 \\
\hline Dist. Max. Conc. $(\mathrm{m})$ & 141,76 \\
\hline
\end{tabular}

Tabla 8 Análisis de dispersión para el monóxido de carbono (CO), combustible fuelóleo. (Fuente elaboración propia)

ISSN: 07 I 8-2724. (http://www.jotmi.org)

Journal of Technology Management \& Innovation (c) Universidad Alberto Hurtado, Facultad de Economía y Negocios. 
ser reutilizadas a través de la Empresa de Recuperación de Materias Primas.

-Los valores de concentración de $\mathrm{CO}$ y SO2 según el aporte del sistema de generador de vapor están por debajo de las concentraciones máximas establecidas por la norma 391999.

-El 100\% del agua de rechazo de la ósmosis inversa que son $7344 \mathrm{~m} 3$ al año puede ser utilizada para el riego de áreas verdes.

3. Se efectuó un análisis económico de las opciones de PML en el cual se demuestra, que el centro ahorraría 174492,8 CUC y 7III CUP al año por concepto de disminución de consumo.

4. La futura implantación de las opciones de PML en el proceso de la EPO-hr conllevaría a disminuir los impactos negativos medioambientales del proceso productivo y sus sistemas auxiliares.

\begin{tabular}{|l|l|l|l|l|}
\hline Partidas & Costo (MN) & Costo (USD) & Ahorro /año (MN) & Ahorro/ año (USD) \\
\hline Recuperación del agua de la ósmosis inversa. & - & - & $\$ 5875$ & - \\
\hline Recuperación de los residuos sólidos & - & - & $\$ 1236$ & - \\
\hline Caracterización de los residuales líquidos & $\$ 10600$ & - & - & - \\
\hline $\begin{array}{l}\text { Cambio de las columnas cromatográficas } \\
\text { G-25 y Superdex por filtros tangenciales }\end{array}$ & - & - & & $\$ 174492,8$ \\
\hline Totales & $\$ 10600$ & - & $\$ 7111$ & $\$ 174492,82$ \\
\hline
\end{tabular}

Tabla 9 Análisis de dispersión para el dióxido de azufre (SO2), combustible fuelóleo (Fuente elaboración propia)

\begin{tabular}{|l|l|}
\hline Parámetros & Contamin \\
\hline $\mathrm{I} 2 / 03 / 20 \mathrm{I0}$ & \\
\hline $\mathrm{Cm}\left(\mu \mathrm{g} / \mathrm{m}^{3}\right)$ & 105,27 \\
\hline Cma $\left(\mu \mathrm{g} / \mathrm{m}^{3}\right)$ & 500 \\
\hline Dist. Max. Conc. $(\mathrm{m})$ & 156,8 \\
\hline $\mathrm{I} 6 / 04 / 2010$ & \\
\hline Cm $\left(\mu \mathrm{g} / \mathrm{m}^{3}\right)$ & 107,45 \\
\hline Cma $\left(\mu \mathrm{g} / \mathrm{m}^{3}\right)$ & 500 \\
\hline Dist. Max. Conc. $(\mathrm{m})$ & 160,95 \\
\hline $8 / 06 / 2010$ & \\
\hline Cm $\left(\mu \mathrm{g} / \mathrm{m}^{3}\right)$ & 125,84 \\
\hline Cma $\left(\mu \mathrm{g} / \mathrm{m}^{3}\right)$ & 500 \\
\hline Dist. Max. Conc. $(\mathrm{m})$ & 141,76 \\
\hline
\end{tabular}

Tabla 10 Costos y ahorros de las opciones PML en cada partida. (Fuente elaboración propia)

ISSN: 07 I8-2724. (http://www.jotmi.org)

Journal of Technology Management \& Innovation (C) Universidad Alberto Hurtado, Facultad de Economía y Negocios. 


\section{Referencias}

APHA-AWWA-WPCS-WEF. (2000) Standard method for the examination of water and wastewater. 2I ST Edition.

CÁRDENAS, L., y Tamayo, J. (2010) Subrama 08.03.04 Recuperación de materias primas. Listado oficial de precios. La Habana.

CLEANER Production UNIDO Tool Kit. (2004) Training Workshop on Cleaner production. Jagüey Grande.

ECO Waste Solutions. Process. (2010) http://www.ecosolutions.com/index.php/screen/español. [Accessed March 8, 2010]

LA O LEYVA, Rogelio. (20II) Estudio de las emisiones de gases contaminantes de la CTE Habana del Este usando crudo nacional. La Habana.

LOBATO. R. (2002) Calidad del agua para riego y su uso en la agricultura. Universidad de Magdalena: Facultad de Ingeniería de Recursos Naturales.

MONTERO, A et al (2002) IA 6746/I5 Aguas naturales. Determinación de $\mathrm{Fe}, \mathrm{Co}, \mathrm{Ni}, \mathrm{Cu}, \mathrm{Zn}$ y Pb. La Habana.

NORMA Cubana 39. Calidad del Aire. Requisitos Higiénicos Sanitarios. (1999) Instituto de investigación de normalización de Cuba.

NORMA Técnica: Procedimientos para el manejo de residuos sólidos hospitalarios: Lima. (2004) Ministerio de Salud.

POZO, J. (2009) Diagnóstico ambiental del agua residual en el Instituto Superior Politécnico José Antonio Echeverría y propuesta de sistemas de tratamiento. Facultad de Química. La Habana.

REINOSA, M. (2013) Obtención de factores de emisión en la Industria Farmacéutica cubana. Instituto Superior Politécnico José Antonio Echeverría. Facultad de Química. La Habana.

RESIDUOS de servicios de salud: Brasil. (1994) Asociación Brasileña de Normas Técnicas.

RESOLUCIÓN I36.09: Desechos Peligrosos. (2009) Ministerio de Ciencia, Tecnología y Medio Ambiente de Cuba.

SYANEZ, I. (2009) El reciclaje de los residuos sólidos plásticos como alternativa para mejorar la calidad ambiental en el municipio de Morelia. Michoacán.
WIKIPEDIA. (2009) Calidad de agua para el uso agrícola. http://es.wikipedia.org/wiki/Calidad_del_agua\#Calidad_ del_Agua_para_uso_Agr.C3.ADcola [Consultado Junio 3, 2009]

ZAROR, C. (2000) Introducción a la ingeniería ambiental para la industria de procesos. Chile. 